\title{
SPARKing: Sampling planning after the results are known
}

\author{
Kyoshiro Sasaki ${ }^{1 \#^{*}}$ and Yuki Yamada ${ }^{2 \#^{*}}$ \\ *These authors contributed equally \\ ${ }^{1}$ Kansai University \\ ${ }^{2}$ Kyushu University
}

\#Corresponding authors

Kyoshiro Sasaki, Ph. D.

Address: Faculty of Informatics, Kansai University,

2-1-1, Ryozenji-cho, Takatsuki, Osaka, 569-1095, Japan.

E-mail: kyoshiro0920@gmail.com

TEL: +81-72-690-2447

Yuki Yamada, Ph. D.

Address: Faculty of Arts and Science, Kyushu University,

744 Motooka, Nishi-ku, 169-8555, Fukuoka, 819-0395, Japan.

E-mail: yamadayuk@gmail.com

TEL: +81-92-802-5837 


\begin{abstract}
In null hypothesis significance testing, it is important how sample size has been determined (i.e., sample size justification) before data collection. Here we introduce a new questionable research practice related to sample size justification: Sampling planning after the results are known (SPARKing). This paper shows two representative cases of SPARKing and discusses solutions to it; however, we think that effective countermeasures are almost non-existent at this time. The main aim of this paper is to introduce SPARKing to the scientific community and to stimulate discussion.
\end{abstract}




\section{SPARKing: Sampling planning after the results are known}

Most psychological research using experimental approaches with null hypothesis significance testing assumes a population, performs sample selection from it, and conducts statistical tests on the sample data. How sample sizes are determined before experiments is highly important for discussion about inferential goals of research through statistical hypothesis testing. Researchers should determine the sample size based on some justifications mostly before collecting the data and should discuss the results depending on the way of the sample size justifications (e.g., Lakens, in press). Moreover, based on the sample size justifications, readers will evaluate how informative the results are. The sample size justifications lead to what can be claimed from the results, and eventually to the reliability of the study. On the other hand, there may be studies that have not justified (or could not justify) the sample size; Honestly reported, no sample size justification is not necessarily bad (Lakens, in press). However, the sample size justification is often required threateningly by submission guidelines or reviewer's comments, and then the manuscript is dismissed (i.e., rejected) simply because of no justification. Given this situation, there may be cases in which the sample size is justified retrospectively after collecting the data: Sampling planning after the results are known (SPARKing). SPARKing possibly hurts credibility in the design of the experiment, which means that it prevents appropriate interpretation and reproducibility of the results of that experiment. This paper introduces the cases when SPARKing is questionable and proposes possible solutions.

\section{When SPARKing is questionable}

\section{Defending significant results already obtained with a small sample size}

Suppose, for example, that a researcher has conducted a direct replication experiment to examine the finding of a previous study that showed the between-participants difference on weight perception between linguistic labels A ("heavy") and B ("light") on the surface of an object to be lifted. After they recruited participants from their pool without determining a specific sample size, 20 participants came at a convenient time and were assigned to each group ( $N=10$ per group). At this time, the researcher analyzed the data, the results were desirable for them, and then they reported the results in a paper titled "A failed replication of the effect of linguistic labels on weight perception" because they did not obtain a significant difference of $t(17.9)=1.09, p=.29$, Cohen's $d=0.49$. However, unfortunately during the peer review process, reviewers pointed out that they did not state any sample size justification, which was required by a submission guideline; along with other reasons, the manuscript was rejected by the first journal. They decided to use SPARKing before submitting to the next journal in order to increase the possibility of acceptance. In the manuscript, they created a new section on "Sample Size Design" and declared the following: We chose 10 participants per group because previous studies on weight perception have traditionally used such a sample size (e.g., Amazeen $\&$ Turvey, 1996; Marsden, Rothwell, \& Traub, 1979), and because we could not involve a large number of participants in the laboratory due to the lockdown caused by the spread of COVID-19. In addition, just to be safe, they registered a protocol with Open Science Framework describing this sample size 
determination, and included its URL in the same named section of the manuscript (i.e., Sample Size Design). As a result, they were successfully able to publish their paper in the next journal without any reviewers pointing out the sample size. Congratulations!

As we see, nothing essential about the sampling has changed in this example, yet only its superficial credibility has been adjusted by SPARKing: The sample size was justified by the other reasons although it was obtained by the stopping rule based on researcher's convenience (i.e., the results supported their idea). Here they used two of the justification patterns listed by Lakens (in press) called "Resource constraints" and "Heuristics". The previous studies they cited were very selectively collected cases, and the sample size of weight perception studies actually varies widely. However, many readers may have actually experienced the similar thing in their own work as well, especially under COVID-19 situations.

\section{Concealing the process of making significant results by increasing $N$}

Suppose that the researcher in Case 1 has conducted a new experiment to examine a correlation between the perceived heaviness of a coin $(¥ 500)$ and the mental arithmetic performance. They decided to perform a prior power analysis this time and first calculated the results with a medium effect size $(r$ $=.3$ ) and $1-\beta=.80$, as they could not predict the effect size, and a one-tailed test as they expected a positive correlation, resulting in a required sample size of 64 participants. They collected data on this number of participants, but unfortunately the results were not significant ( $p=.08$ ). They could have discontinued data collection at this point, assuming that a significant trend (or marginally significant results) had been obtained, but the results appeared weak to publish, and they had a feeling that the correlation might become significant if they increased $N$ a little more, so they collected more data. They reasoned among themselves that "in fact, the direction of the predicted relationship was not known a priori," so they changed the test to two-tailed and reperformed a power analysis, which resulted in the required sample size of 82 participants, so they added data up to that point, which was again regrettably not significant $(p=.07)$. They then changed the test back to one-tailed, set the 1- $\beta$ to .90 , and did the power analysis again, which required 88 participants. They did the same thing again, with the similar trend $(p=.07)$. They continued to finely tune the effect size, power, and direction of the test for various reasons, and when they finally increased the data to 614 participants with $r=.1,1$ $\beta=.80$, and a one-tailed setting, they found a significant correlation $(p=.04)$. Fortunately, it was an online survey using crowdsourcing, and thus it was not so difficult for them to increase the sample size. They pre-registered with this sample size design, and in the same named section of the manuscript described only this last setting with a "good" reason, and then the paper was successfully published, congratulations!

Here, $p$-hacking by increasing $N$ was used (Simmons, Nelson, \& Simonsohn, 2011) while providing justification. When the sample size is increased and multiple tests are performed, significant results can almost always be obtained, even with data drawn randomly (Albers, 2019). They did this by trying many possible settings in succession within a justifiable range, stopping the addition of data 
at the point where significant results were obtained, and reporting only the final results and settings. Yet for many readers, this is not the first time hearing about it as well.

Note that in common with both cases a questionable research practice (QRP) dedicated to preregistration called PARKing was also used. PARKing is an abbreviation for pre-registration after the results are known, and it is an act that severely diminishes the meaning of pre-registration and attempts to gain only superficial credibility (Parsons et al., 2022; Yamada, 2018). SPARKing and PARKing work very well together because sample size design is always done in pre-registration. This is a very bad misuse of the pre-registration system, because it is a false representation of the plan post hoc. Moreover, of course, it is perfectly acceptable to "add a description" of sample size justification to the manuscript after submission, if necessary, in the case that it was actually decided in advance. What we are concerned with here is whether it was actually decided in advance, and whether the reader can detect it. SPARKing is the practice of pretending to have justified a sample size when in fact it has not been justified, and writing sample size justification additionally in the manuscript itself is not a problem.

\section{Solutions?}

How can we detect SPARKing? First of all, it is strongly hoped that authors will not do this QRP; as Lakens (in press) noted, information of "no justification" (or even if it is just "conveniency") is also useful for readers. If authors do not have a specific and legitimate prior design, they should state it honestly.

Then, when we cannot expect authors to do such honest research practices, what should we do? First, if questionable manuscripts or papers have the corresponding preprints, then it can be checked whether there are differences in sample size justifications between them; however, this way is meaningless when no corresponding preprint exists, it has been uploaded after disguise, or a researcher just has added a description of sample size justification because of lefting it out initially. Second, in the case of using SPARKing in conjunction with PARKing (Parsons et al., 2022; Yamada, 2018), differences in timestamps between pre-registrations and all the files of unprocessed data in a repository might be helpful to guess these QRPs. However, the files of unprocessed data are unavailable or the timestamps of the files have been forged or tampered by some ways (e.g., changing the system date for the operating system), this way is inefficient, too. Thus, detecting SPARKing is difficult at this time. Because it is possible that the studies, for which SPARKing is taken, have odd sample size and effect size in light of domain knowledge, readers including reviewers and editors should carefully interpret the effects reported in these studies; even if SPARKing and other QRPs are not taken, these effects possibly include false negatives and false positives when merely focusing on $p$-values.

However, there seems to be a way to deter SPARKing. Peer-reviewed pre-registration (i.e., the Registered Reports system: e.g., Nosek, Ebersole, DeHaven, \& Mellor, 2018; Nosek \& Lakens, 2014) is possibly the promising solution. Under this system, a manuscript containing only protocols 
(i.e., protocol manuscript) is peer-reviewed before data collection, and researchers can collect the data after the protocol manuscript has passed the peer-review. Importantly, researchers have to conduct their experiments and data analyses in line with the accepted protocol manuscript. Of course, the sample size justifications are required in the protocol manuscript and cannot be changed after it is accepted, and thus there is no way SPARKing could happen. Considering that the Registered Reports system is also a countermeasure for PARKing (Ikeda, Xu, Fuji, Zhu, \& Yamada, 2019; Yamada, 2018), it is a strong prevention method.

Perhaps some other possible solutions we are not aware of could be found. If someone comments on a useful idea using the Hypothes.is service available on PsyArXiv, we would like to invite them to write the part with us as co-authors. 


\section{Acknowledgement}

We would like to thank Dr. Hiroyuki Muto for the valuable comments on statistical mistakes and other issues in revising the manuscript. He also pointed out important concerns that our discussion about SPARKing possibly leads to unfairly discounting the value of sound research by questioning it. While our position is that it is important to bring potential problems to light and discuss them, at the same time we can understand his opinion. 


\section{References}

Albers, C. (2019). The problem with unadjusted multiple and sequential statistical testing. Nature Communications, 10, 1921. https://doi.org/10.1038/s41467-019-09941-0

Amazeen, E. L., \& Turvey, M. T. (1996). Weight perception and the haptic size-weight illusion are functions of the inertia tensor. Journal of Experimental Psychology: Human Perception and Performance, 22(1), 213-232. https://doi.org/10.1037//0096-1523.22.1.213

Ikeda, A., Xu, H., Fuji, N., Zhu, S., \& Yamada, Y. (2019). Questionable research practices following pre-registration. Japanese Psychological Review, 62(3), 281-295. https://doi.org/10.24602/sjpr.62.3_281

Lakens, D. (in press). Sample Size Justification. Collabra: Psychology. (Preprint: https://doi.org/10.31234/osf.io/9d3yf)

Marsden, C. D., Rothwell, J. C., \& Traub, M. M. (1979). Effect of thumb anaesthesia on weight perception, muscle activity and the stretch reflex in man. The Journal of Physiology, 294, 303315. https://doi.org/10.1113/jphysiol.1979.sp012931

Nosek, B. A., Ebersole, C. R., DeHaven, A. C., \& Mellor, D. T. (2018). The preregistration revolution. Proceedings of the National Academy of Sciences, 115, 2600-2606. https://doi.org/10.1073/pnas.1708274114

Nosek, B. A., \& Lakens, D. (2014). Registered reports: a method to increase the credibility of published results. Social Psychology, 45, 137-141. http://doi.org/10.1027/1864-9335/a000192

Parsons, S., Azevedo, F., Elsherif, M.M., Guay, S., Shahimet, O.N., Govaart, G...Aczel, B. (2022). A community-sourced glossary of open scholarship terms. Nature Human Behaviour. https://doi.org/10.1038/s41562-021-01269-4

Simmons, J. P., Nelson, L. D., \& Simonsohn, U. (2011). False-positive psychology: Undisclosed flexibility in data collection and analysis allows presenting anything as significant. Psychological Science, 22(11), 1359-1366. https://doi.org/10.1177/0956797611417632

Yamada, Y. (2018). How to crack pre-registration: Toward transparent and open science. Frontiers in Psychology, 9:1831. https://doi.org/10.3389/fpsyg.2018.01831 


\section{Funding}

The present study was supported by JSPS KAKENHI Grant Numbers JP19K14482 (K.S.), JP21H03784 (K.S. and Y.Y.), JP20H04581 (Y.Y.), JP18K12015 (Y.Y.), JP17H00875 (Y.Y.), and JP16H03079 (Y.Y.). 
Declaration of interests statement

The authors declare no conflict of interest. 


\section{Author contributions}

KS: Conceptualization, Funding Acquisition, Project Administration, Resources, Writing-Original Draft, and Writing - Review and Editing.

YY: Conceptualization, Funding Acquisition, Supervision, Resources, Writing-Original Draft, and Writing-Review and Editing. 